\title{
Dynamic changes of five neurotransmitters and their related enzymes in various rat tissues following $\beta$-asarone and levodopa co-administration
}

\author{
LIPING HUANG ${ }^{1,2^{*}}$, MINZHEN DENG $^{1 *}$, YONGQI FANG $^{1}$ and LING LI ${ }^{1}$ \\ ${ }^{1}$ Laboratory Center, The First Affiliated Hospital, Guangzhou University of Chinese Medicine, \\ Guangzhou, Guangdong 510405; ${ }^{2}$ Hainan Medical University, Haikou, Hainan 571199, P.R. China
}

Received September 10, 2014; Accepted July 23, 2015

DOI: $10.3892 / \mathrm{etm} .2015 .2704$

\begin{abstract}
The aim of the present study was to investigate the dynamic changes of five neurotransmitters and their associated enzymes in the rat plasma and brain tissues following the co-administration of $\beta$-asarone and levodopa (L-dopa). The rats were divided into five groups, including the control group and four treatment groups that were intragastrically co-administered $\beta$-asarone and L-dopa and sacrificed at 1, 5, 18 and 48 h, respectively. Neurotransmitter levels in the brain tissues and plasma were detected using high performance liquid chromatograph and the related enzymes of dopamine (DA) were measured using an enzyme-linked immunosorbent assay. The results indicated that the striatal levels of L-dopa and 3,4-dihydroxyphenylacetic acid (DOPAC) peaked at $1 \mathrm{~h}$ and then returned to the normal levels, while the striatal levels of DA were stable within $48 \mathrm{~h}$. In the cortex and hippocampus tissue, L-dopa, DA, DOPAC and homovanillic acid (HVA) levels peaked at $1 \mathrm{~h}$ and then returned to normal levels. In the plasma, L-dopa, DA, DOPAC and HVA levels peaked at 1 h. Compared with the control group, L-dopa, DA and HVA levels were higher between 18 and $48 \mathrm{~h}$, whereas the DOPAC level was lower. By contrast, no statistically significant differences were observed in the serotonin (5-HT) levels among the plasma, hippocampus, cortex and striatum. Furthermore, the DA/L-dopa ratio in the brain tissues and plasma increased in the first $5 \mathrm{~h}$, while (DOPAC + HVA)/DA ratios demonstrated a significant reduction. Striatal tyrosine hydroxylase $(\mathrm{TH})$ and aromatic amino acid decarboxylase (AADC) levels were higher compared with the control group; however,
\end{abstract}

Correspondence to: Associate Professor Ling Li, Laboratory Center, The First Affiliated Hospital of Guangzhou University of Chinese Medicine, 12 Jichang Road, Guangzhou, Guangdong 510405, P.R. China

E-mail: liling88800@126.com

*Contributed equally

Key words: neurotransmitter, $\beta$-asarone, levodopa, dopamine, aromatic amino acid decarboxylase, catechol- $O$-methyltransferase
catechol-O-methyltransferase (COMT) and monoamine oxidase B levels were reduced. In the rat plasma, TH and COMT peaked at $1 \mathrm{~h}$, while AADC peaked at $5 \mathrm{~h}$. In conclusion, the results of the present study indicate that the co-administration of L-dopa and $\beta$-asarone may be used to maintain a stable striatal DA level within $48 \mathrm{~h}$. In addition, this treatment may promote DA generation by AADC and reduce the metabolism of DA by COMT.

\section{Introduction}

Parkinson's disease (PD) is predominantly caused by the death of dopaminergic neurons in the substantia nigra, and results in deficient striatal dopamine (DA) levels, which are responsible for the motor symptoms of PD, including bradykinesia, tremor and rigidity (1). Currently, the primary treatment for PD is supplemental DA therapy (2). Tyrosine hydroxylase (TH) is a rate-limiting enzyme of DA synthesis, which catalyzes the hydroxylation of tyrosine to levodopa (L-dopa) (3). L-dopa is subsequently converted into DA by aromatic amino acid decarboxylase (AADC) (4). In addition, DA is converted into 3,4-dihydroxyphenylacetic acid (DOPAC) by monoamine oxidase B (MAO-B) (5), and into homovanillic acid (HVA) by catechol- $O$-methyltransferase (COMT) (6). Therefore, L-dopa, DA, DOPAC, HVA, AADC, MAO-B, COMT and TH may be implicated in the pathogenesis of PD.

It has been demonstrated that L-dopa is the most effective constituent of typical PD treatment (7). However, the long-term use of L-dopa is associated with severe side effects, including motor response fluctuations and the emergence of drug-induced involuntary movements (8). For instance, Madopar, which consists of L-dopa and benserazide, is the firstline treatment option for PD. Long-term administration of Madopar is complicated by the development of various types of motor response oscillation, as well as drug-induced dyskinesia, which is a complication characterized by erratic involuntary movements, hypotension and psychiatric symptoms (9). Hence, Madopar does not resolve the adverse effects of L-dopa.

$\beta$-asarone (cis-2,4,5-Trimethoxy-1-propenylbenzene) is a strong fat-soluble substance with a low molecular weight ( $208 \mathrm{~g} / \mathrm{mol}$ ), which is able to rapidly traverse the blood-brain 
barrier, with a peak traversal time of $12 \mathrm{~min}$, and has a half-life of $54 \mathrm{~min}$ (10). Our previous experiments indicated that $\beta$-asarone has a wide range of pharmacological effects on the central nervous system (CNS) and may be widely distributed in the rat hippocampus and cortex (11). Notably, we demonstrated that $\beta$-asarone and L-dopa co-administration was able to significantly increase the striatal levels of DA in healthy rat tissue (11). In addition, the striatum, hippocampus and cortex were the three important parts of the CNS (12). However, to the best of our knowledge, there are no prior studies that describe the effects of $\beta$-asarone and L-dopa co-administration on the dynamic changes of neurotransmitters in the rat striatum, cortex, hippocampus and plasma.

Therefore, the aim of the present study was to investigate the dynamic changes in the levels of L-dopa, DA, DOPAC, HVA and serotonin (5-HT) in the plasma, striatum, hippocampus and cortex of healthy rats following $\beta$-asarone and L-dopa co-administration, using high-performance liquid chromatography (HPLC) and fluorescence detection (FD). Furthermore, in order to observe the dynamic changes in the levels TH, COMT, AADC and MAO-B after co-administration within $48 \mathrm{~h}$, the levels of these enzymes in the plasma and striatum were evaluated using an enzyme-linked immunosorbent assay (ELISA).

\section{Materials and methods}

Experimental design. A total of 40 Sprague Dawley rats (20 female and 20 male; weight, 220-250 g) were obtained from the Laboratory Animal Center of Guangzhou University of Chinese Medicine (ethical code no. TCMF1-2012028; Guangzhou, China). The animals were housed in a light- and temperature-controlled room with free access to standard food and water. The experimental protocols were approved by the Ethics Committee of Guangzhou University of Chinese Medicine and were consistent with the international guidelines.

Rats were divided into five groups ( $n=8$ per group): Control group and four groups that received a single co-administration of $\beta$-asarone and L-dopa, at 15 and $60 \mathrm{mg} / \mathrm{kg}$ body weight, respectively. The rats in the four treatment group rats were subsequently sacrificed by cervical dislocation at 1, 5, 18 and $48 \mathrm{~h}$, respectively. The control rats received an equal volume of normal saline vehicle, following the same procedure and were sacrificed at $48 \mathrm{~h}$.

Preparation of $\beta$-asarone. The $\beta$-asarone used in this study was extracted from Acorus tatarinowii Schott according to a previously-reported procedure (13). The purity of $\beta$-asarone was $\sim 99.55 \%$ (14), which was confirmed by gas chromatography-mass spectrometry, infrared spectrum and nuclear magnetic resonance detection, which was conducted at the China National Analytical Center (Guangzhou, China).

Sample collection. Following the co-administration, rats were anesthetized with $10 \%$ chloral hydrate $(3.5 \mathrm{mg} / \mathrm{kg}$, intraperitoneal injection) at the pre-specified times of $1,5,18$ and $48 \mathrm{~h}$ after treatment. Next, the limbs of the anesthetized rats were fixed on an autopsy table and the rat hearts were exposed in the thoracic cavity by opening the abdominal cavity that is below the xiphoids. Blood samples were collected from the aortic artery, and the plasma was separated by centrifugation at $3,000 \mathrm{x} \mathrm{g}$ for $10 \mathrm{~min}$ and stored at $-80^{\circ} \mathrm{C}$ for subsequent HPLC analysis. Subsequently, in order to remove the blood from the rat brain, normal saline was perfused into the left ventricle and evacuated from the right atrial appendage. The perfusion was discontinued when the rat eyes and claws turned pale. The striatum, hippocampus and cortex areas were then dissected rapidly from the brains on ice and stored at $-80^{\circ} \mathrm{C}$ for HPLC analysis. Sample collection from the control group rats was performed following the same procedure.

HPLC analysis of DA, L-dopa, DOPAC, HVA and 5-HT. Briefly, the striatum, hippocampus and cortex were weighed and homogenized in ice-cold $0.1 \mathrm{M}$ perchloric acid $(1: 5 \mathrm{~g} / \mathrm{ml})$ by sonication at $40 \mathrm{kHz}$ for $5 \mathrm{~min}$. Homogenates were centrifuged at $12,000 \mathrm{x} \mathrm{g}$ for $15 \mathrm{~min}$ at $4^{\circ} \mathrm{C}$, then the supernatants were collected and filtered through microporous membrane filters $(0.22 \mu \mathrm{m})$, and $20 \mu \mathrm{l}$ of each sample was injected into the HPLC column. In addition, $0.1 \mathrm{M} \mathrm{HClO}_{4}$ was added to $500 \mu \mathrm{l}$ plasma at a ratio of 1:1 (v/v). The mixture was subjected to vortex mixing and centrifugation at $13,000 \mathrm{x}$ g for $15 \mathrm{~min}$ at $4^{\circ} \mathrm{C}$. Next, the supernatants were collected and filtered through microporous membrane filters $(0.22 \mu \mathrm{m})$, and $20 \mu \mathrm{l}$ of each sample was subjected to HPLC. The control substances of DA, 5-HT and L-dopa were obtained from National Institutes for Food and Drug Control (Beijing, China), while DOPAC and HVA were purchased from Sigma-Aldrich (St. Louis, MO, USA).

The concentrations of DA and L-dopa were quantified by HPLC and FD, using a Waters 2695 separations module and a Waters 2475 multiwavelength fluorescence detector (Waters Corporation, Milford, MA, USA) at an excitation and absorption wavelength of 280 and $330 \mathrm{~nm}$, respectively. Separation was performed using a 5- $\mu$ m Hypersil $^{\mathrm{TM}}$ ODS2 column (150x4.6 mm; Dalian Elite Analytical Instruments Co., Ltd., Dalian, China) with column temperature of $30^{\circ} \mathrm{C}$ and a flow rate of $1 \mathrm{ml} / \mathrm{min}$. The mobile phase was composed of $0.1 \mathrm{M} \mathrm{KH}_{2} \mathrm{PO}_{4}$ and methanol. The column was equilibrated with mobile phase for $30 \mathrm{~min}$ prior to analysis. This method was validated for the determination of neurotransmitter levels as reported in our previous study (15). Data were analyzed using Empower 2 chromatography data software (Waters Corporation) and the results were calculated and expressed as $\mu \mathrm{g} / \mathrm{g}$ and $\mu \mathrm{g} / \mathrm{ml}$ for tissue and plasma, respectively.

TH, COMT, AADC and MAO-B analyses. The striatum was weighed and homogenized with ice-cold normal saline $(1: 3 \mu \mathrm{l} / \mathrm{mg})$, and then centrifuged at $3,000 \mathrm{x} \mathrm{g}$ for $10 \mathrm{~min}$ to obtain the supernatant. TH (T031FC), COMT (C033FC), AADC (A036FC) and MAO-B (M035FC) were determined separately using ELISA kits (Shanghai Saimo Biotechnology Co., Ltd., Shanghai, China) and an American Hyperion MRIII microplate reader (BioTek Instruments, Inc., Winooski, VT, USA) according to the manufacturer's instructions. In addition, ELISA kits from the same supplier were used to separately determine the plasma levels of AADC (A036SC), COMT (C033SC) and TH (T031SC).

Statistical analysis. Data are expressed as the mean \pm standard deviation and statistical differences between groups 
A

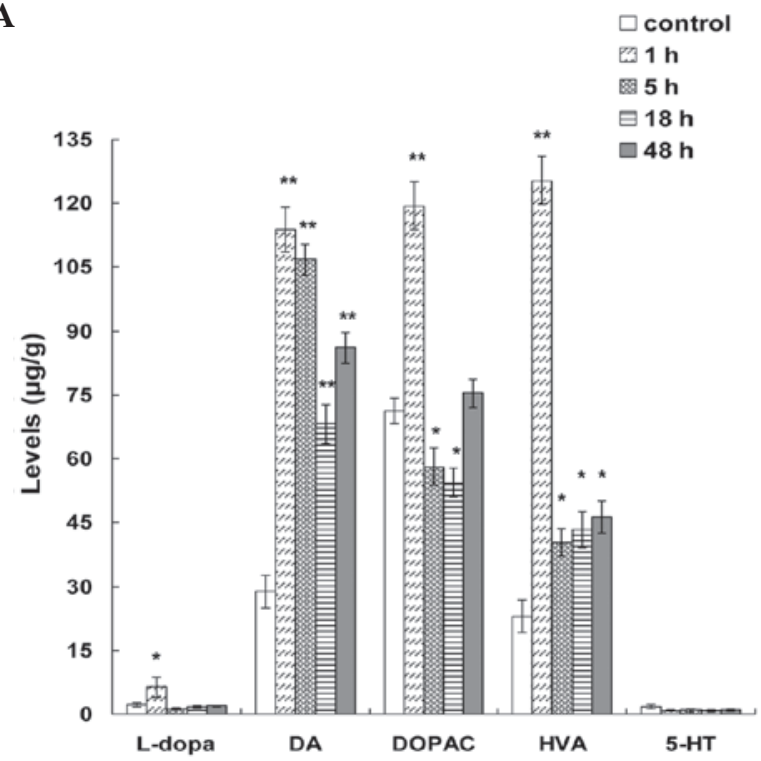

Neurotransmitters in striatum of normal rats

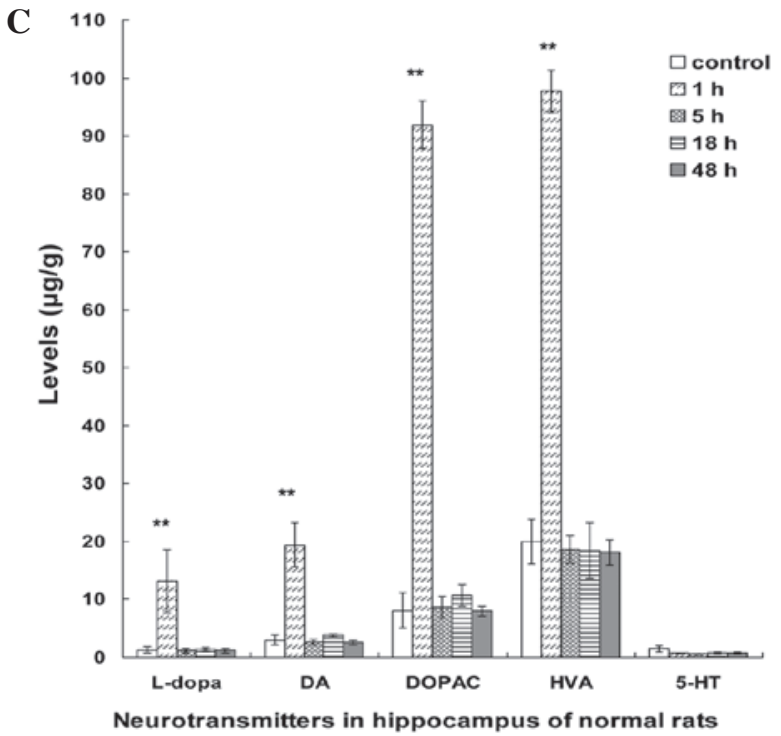

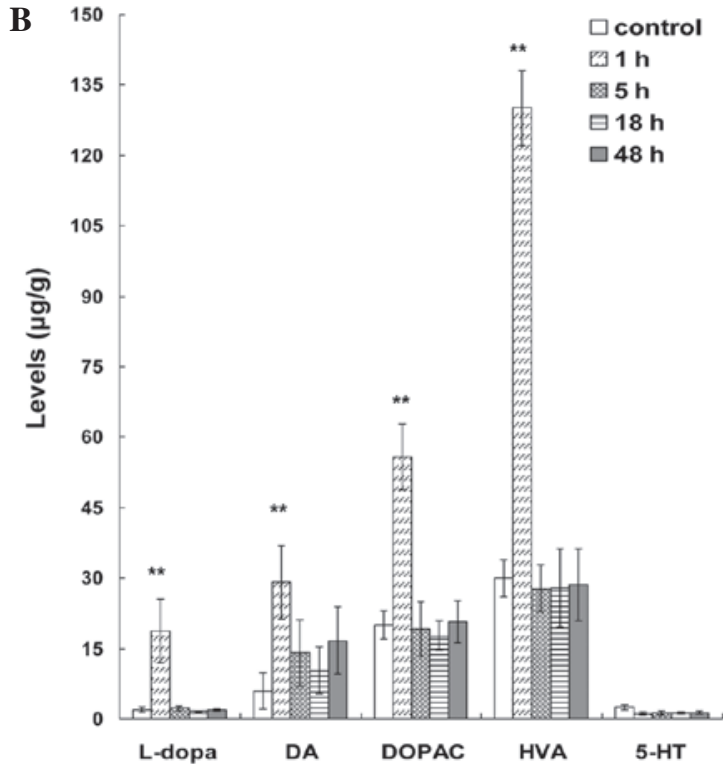

Neurotransmitters in cortex of normal rats

D

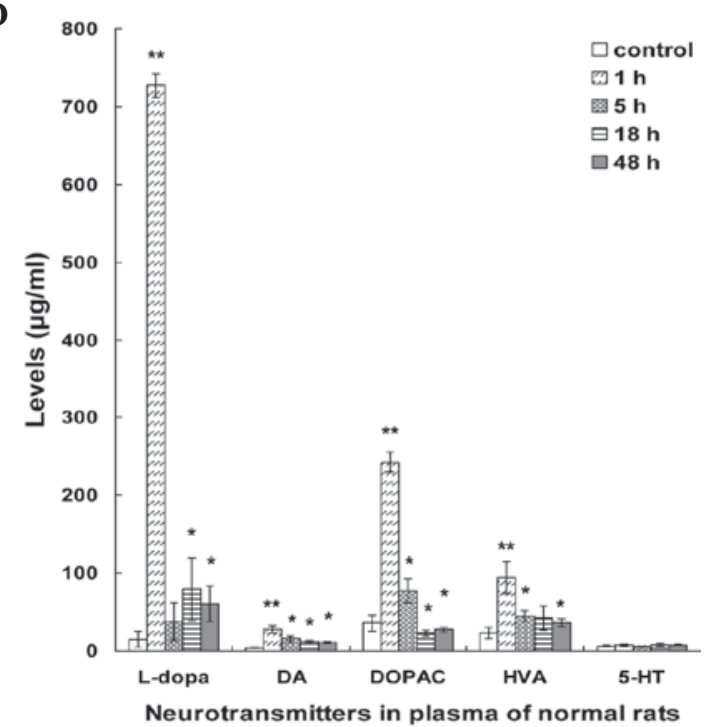

Figure 1. Dynamic changes in the levels of the neurotransmitters, L-dopa, DA, DOPAC, HVA and 5-HT, in the plasma and brain tissues of healthy rats at various time points after the co-administration of $\beta$-asarone and L-dopa. Differences in the levels of the five neurotransmitters in the (A) striatum, (B) cortex, (C) hippocampus and (D) plasma of the rats within $48 \mathrm{~h}\left(\mu \mathrm{g} / \mathrm{g}\right.$ tissue weight) are presented. Bars represent the mean \pm standard deviation of 8 rats. ${ }^{*} \mathrm{P}<0.05$ and ${ }^{* *} \mathrm{P}<0.01$ vs. control group (analysis of variance with Bonferroni test). L-dopa, levodopa; DA, dopamine; DOPAC, 3,4-dihydroxyphenylacetic acid; HVA, homovanillic acid; 5-HT, serotonin.

were determined by one-way analysis of variance followed by Bonferroni post-hoc test for multiple comparisons at $\mathrm{P}<0.05$. $\mathrm{P}<0.05$ was considered to indicate a statistically significant difference. Correlations between the neurotransmitters were performed by Pearson correlation. All the statistical analyses were performed using SPSS statistical software, version 17.0 (SPSS, Inc., Chicago, IL, USA).

\section{Results}

Levels of the five neurotransmitters changed in the rat plasma, hippocampus, cortex and striatum following treatment. Subsequent to co-administration of $\beta$-asarone and L-dopa, the L-dopa levels increased in the striatum of the rats, peaking at $1 \mathrm{~h}$, then deceasing markedly at $5 \mathrm{~h}$ and remaining stable between 5 and $48 \mathrm{~h}$. Compared with the control group, L-dopa levels increased significantly in the $1 \mathrm{~h}$ group $(\mathrm{P}<0.05)$. DA levels also increased and peaked at $1 \mathrm{~h}$, showing a slight reduction at $5 \mathrm{~h}$, followed by a further reduction between 5 and $18 \mathrm{~h}$ and a subsequent increase. DA levels demonstrated a significant increase in the 1, 5, 18 and $48 \mathrm{~h}$ groups compared with the control group $(\mathrm{P}<0.05)$. Similarly, DOPAC levels increased significantly in the $1 \mathrm{~h}$ group compared with the control group $(\mathrm{P}<0.01)$, decreased linearly between 5 and $18 \mathrm{~h}(\mathrm{P}<0.05)$, and then exhibited a gradual increase in the $48 \mathrm{~h}$ group. In addition, HVA levels peaked at $1 \mathrm{~h}$, then declined markedly at $5 \mathrm{~h}$ and increased slightly between 5 and $48 \mathrm{~h}$. Compared to the control group, HVA levels demonstrated a significant increase in all the treatment groups $(1,5,18$ and $48 \mathrm{~h} ; \mathrm{P}<0.05)$. By contrast, 
A

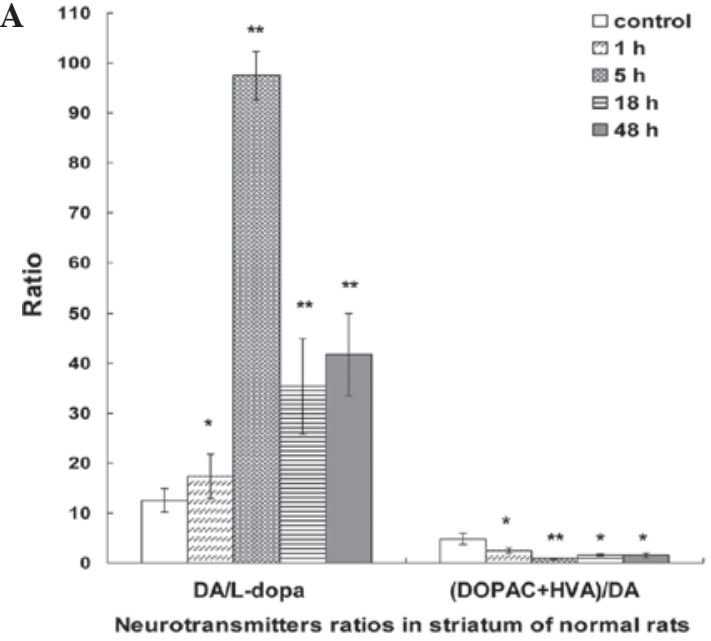

C

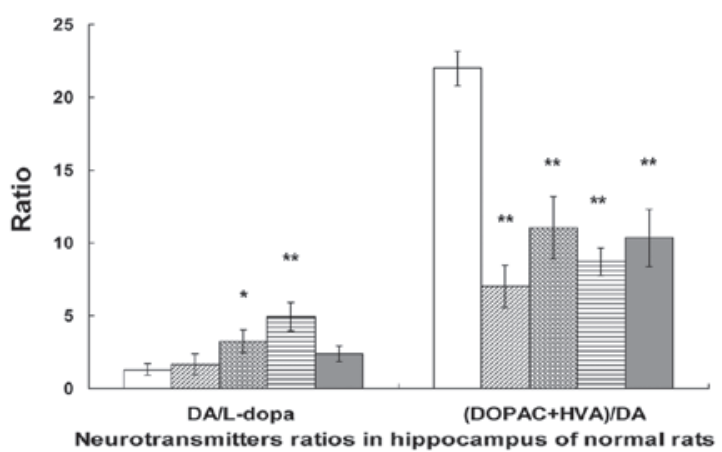

$\square$ control

๒1 h

间 5

$\boxminus 18 \mathrm{~h}$

$\square 48 \mathrm{~h}$
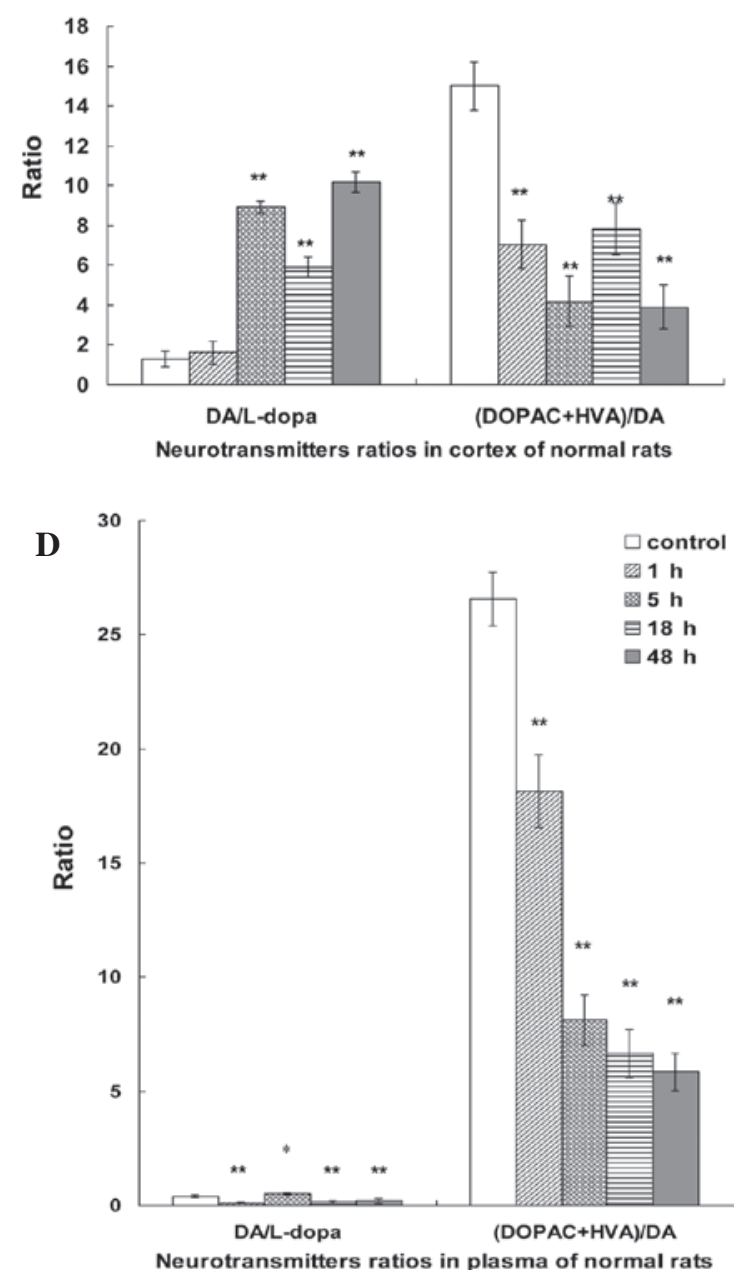

Figure 2. Dynamic changes in the ratios of DA/L-dopa and (DOPAC + HVA)/DA in the plasma and brain tissues of healthy rats at various time points after the co-administration of $\beta$-asarone and L-dopa. The DA/L-dopa and (DOPAC + HVA)/DA ratios in the (A) striatum, (B) cortex, (C) hippocampus and (D) plasma of normal rats are presented. Error bars represent the mean \pm standard deviation of 8 rats. ${ }^{*} \mathrm{P}<0.05$ and ${ }^{* *} \mathrm{P}<0.01$ vs. control group (analysis of variance with Bonferroni test). L-dopa, levodopa; DA, dopamine; DOPAC, 3,4-dihydroxyphenylacetic acid; HVA, homovanillic acid.

5-HT levels remained stable between 1 to $48 \mathrm{~h}$ and were not significantly different compared with the control group (Fig. 1A).

In the cortex and hippocampus, the levels of L-dopa, DA, DOPAC and HVA peaked at $1 \mathrm{~h}$, followed by a sharp decline at $5 \mathrm{~h}$ and a stable level between 5 and $48 \mathrm{~h}$. Compared with the control group, the levels of L-dopa, DA, DOPAC and HVA were significantly increased in the $1 \mathrm{~h}$ group $(\mathrm{P}<0.01)$; however, the 5-HT levels remained stable between 1 and $48 \mathrm{~h}$ and showed no significant difference compared with that of the control group (Fig. 1B and C).

In the rat plasma, L-dopa levels increased and peaked at $1 \mathrm{~h}$, then decreased markedly at $5 \mathrm{~h}$, increased slightly at $18 \mathrm{~h}$ before finally declining slightly at $48 \mathrm{~h}$. In addition, DA levels increased and peaked at $1 \mathrm{~h}$, and subsequently decreased slightly between 5 and $48 \mathrm{~h}$. In addition, DOPAC levels increased and peaked at $1 \mathrm{~h}$, then showed a sharp decline at $5 \mathrm{~h}$, followed by a further reduction between 18 and $48 \mathrm{~h}$. HVA levels peaked at $1 \mathrm{~h}$, decreased sharply at $5 \mathrm{~h}$, then declined slowly between
18 and 48 h. 5-HT levels remained a stable between 1 and $48 \mathrm{~h}$ and showed no significant difference compared with the control group. Furthermore, the levels of L-dopa, DA, DOPAC and HVA demonstrated a marked increase in the $1 \mathrm{~h}$ group compared with the control group $(\mathrm{P}<0.01$; Fig. 1D).

Comparisons of DA/L-dopa and (DOPAC $+H V A) / D A$ ratios in the plasma, striatum, hippocampus and cortex. In the striatum, the DA/L-dopa ratio showed a significant increase in the initial $5 \mathrm{~h}$, followed by a sharp reduction at $18 \mathrm{~h}$, and then increased slightly at $48 \mathrm{~h}$. However, the (DOPAC $+\mathrm{HVA}) / \mathrm{DA}$ ratio exhibited a marked reduction at $1 \mathrm{~h}$, reaching a minimum at $5 \mathrm{~h}$ and then increasing slightly between 18 and $48 \mathrm{~h}$ (Fig. 2A).

In the cortex, the DA/L-dopa ratio demonstrated a sharp increase in the first $5 \mathrm{~h}$, followed by a rapid decline at $18 \mathrm{~h}$, and then increased significantly at $48 \mathrm{~h}$. By contrast, the $($ DOPAC + HVA $) /$ DA ratio showed a sharp decline in the first $5 \mathrm{~h}$, followed by a marked increase at $18 \mathrm{~h}$, and subsequently decreased at $48 \mathrm{~h}$ (Fig. 2B). 
In the hippocampus, the DA/L-dopa ratio exhibited an increasing tendency in the first $18 \mathrm{~h}$, and then returned to the normal levels. In addition, the (DOPAC + HVA)/DA ratio showed a sharp decline in the first $1 \mathrm{~h}$, followed by a slight increase between 5 and $48 \mathrm{~h}$ (Fig. 2C).

In the plasma, when compared with the control group, the DA/L-dopa ratio showed a significant decline in the first $1 \mathrm{~h}$, and then peaked at $5 \mathrm{~h}$ followed by a further reduction between 18 and 48 h. Furthermore, the (DOPAC + HVA)/DA ratio presented a sharp reduction after $48 \mathrm{~h}$, when compared with the control group (Fig. 2D).

Comparison of DA, L-dopa, DOPAC, HVA and 5-HT alterations in the plasma, striatum, hippocampus and cortex following the co-administration. Following co-administration of $\beta$-asarone and L-dopa, the L-dopa levels increased and peaked at $1 \mathrm{~h}$, followed by a sharp decline at $5 \mathrm{~h}$, and remained stable between 5 and $48 \mathrm{~h}$ in the plasma, cortex, hippocampus and striatum. Among these, the L-dopa levels in the plasma were the highest. Furthermore, DA levels increased and peaked at $1 \mathrm{~h}$ in the plasma, hippocampus, cortex and striatum. Among these, the striatal levels of DA were the highest within $48 \mathrm{~h}$, showing a slight reduction at $5 \mathrm{~h}$, followed by a further reduction at $18 \mathrm{~h}$ and subsequent increase. However, DA levels in the cortex, hippocampus and plasma demonstrated a sharp decline at $5 \mathrm{~h}$, followed by stable levels between 5 and $48 \mathrm{~h}$. In addition, the striatal levels of DOPAC peaked at $1 \mathrm{~h}$, followed by a sharp decline at $5 \mathrm{~h}$, and remained stable until $18 \mathrm{~h}$, prior to increasing at $48 \mathrm{~h}$. DOPAC levels in the cortex, hippocampus and plasma exhibited a rapid decrease at $5 \mathrm{~h}$, followed by a steady level between 5 and $48 \mathrm{~h}$. Furthermore, striatal levels of HVA showed a marked reduction between 1 and $5 \mathrm{~h}$, followed by a gradual increase between 5 and $48 \mathrm{~h}$. Similarly, HVA levels in the cortex, hippocampus and plasma displayed a sharp reduction between 1 and $5 \mathrm{~h}$, but remained stable between 5 and $48 \mathrm{~h}$. By contrast, the 5-HT levels remained stable and showed no statistically significant differences from the control group levels within $48 \mathrm{~h}$ among the plasma, striatum, cortex and hippocampus.

Neurotransmitter ratio alterations in the plasma and brain following the co-administration. The DA/L-dopa ratio in the striatum and cortex showed a marked increase in the first $5 \mathrm{~h}$ after treatment, followed by a sharp reduction at $18 \mathrm{~h}$, and subsequently returned to normal levels. In addition, the DA/L-dopa ratio in the hippocampus exhibited a rapid increase in the first $18 \mathrm{~h}$, followed by a sharp reduction at $48 \mathrm{~h}$. Furthermore, the DA/L-dopa ratio in the plasma increased and peaked at $5 \mathrm{~h}$, followed by a rapid reduction at $18 \mathrm{~h}$, and remained stable between 18 and $48 \mathrm{~h}$.

The (DOPAC + HVA)/DA ratio in the plasma and striatum showed a rapid reduction within $48 \mathrm{~h}$. In addition, the (DOPAC + HVA)/DA ratio in the cortex demonstrated a reduction in the first $5 \mathrm{~h}$, followed by a rapid increase at $18 \mathrm{~h}$. Similarly, the (DOPAC + HVA)/DA ratio in the hippocampus showed a sharp decline at $1 \mathrm{~h}$, followed by a reduction at $18 \mathrm{~h}$, and subsequently returned to normal levels.

Alterations in TH, COMT, AADC and MAO-B levels at various time points. In the striatum, TH levels increased and peaked
A

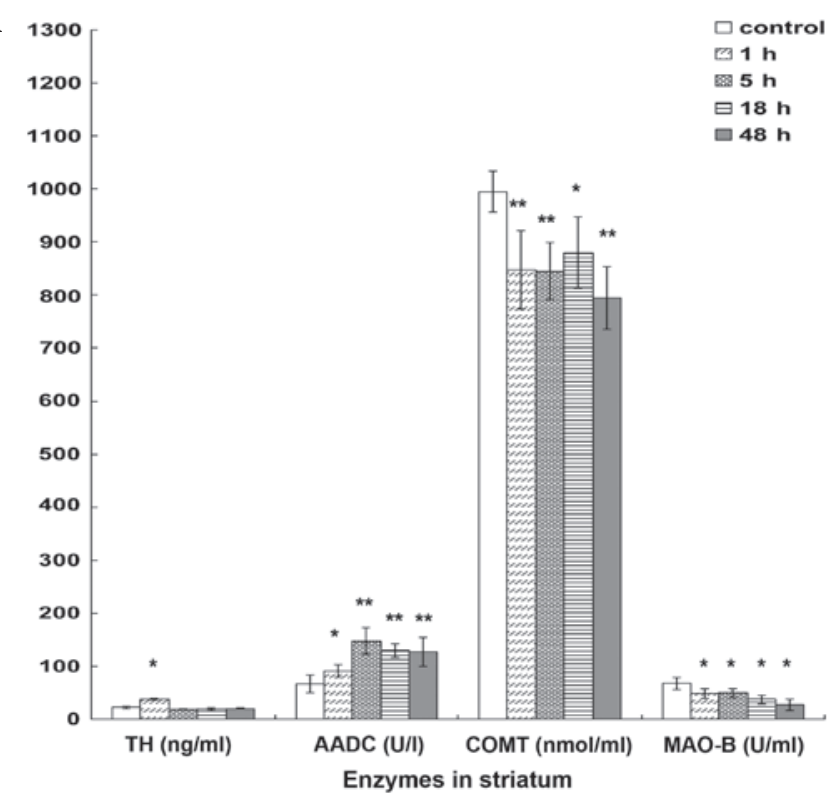

B

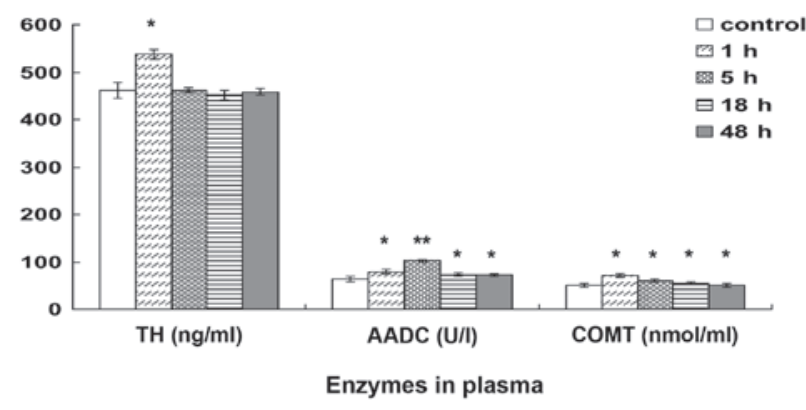

Figure 3. Dynamic changes in the levels of enzymes associated with DA synthesis at various time points after the co-administration of $\beta$-asarone and L-dopa. (A) Changes in the TH, AADC, COMT and MAO-B levels in the striatum of rats. (B) Changes in the TH, AADC and COMT levels in the plasma of the rats. Error bars represent the mean \pm standard deviation of 8 rats. ${ }^{*} \mathrm{P}<0.05$, ${ }^{* *} \mathrm{P}<0.01$ vs. control group (analysis of variance with Bonferroni test). TH, tyrosine hydroxylase; AADC, aromatic amino acid decarboxylase; COMT, catechol- $O$-methyltransferase; MAO-B, monoamine oxidase B.

at $1 \mathrm{~h}$, followed by a marked reduction at $5 \mathrm{~h}$, remaining stable between 5 and $48 \mathrm{~h}$. The AADC levels demonstrated a rapid increase in the first $5 \mathrm{~h}$ followed by a further reduction at $18 \mathrm{~h}$. By contrast, the COMT levels showed a sharp reduction at $1 \mathrm{~h}$, followed by a further reduction at $48 \mathrm{~h}$. In addition, MAO-B levels showed a slight reduction between 1 and $48 \mathrm{~h}$ (Fig. 3A).

In the plasma, $\mathrm{TH}$ levels increased at $1 \mathrm{~h}$, followed by a rapid reduction at $5 \mathrm{~h}$, and remained at a stable level between 5 and $48 \mathrm{~h}$. AADC levels exhibited a gradual increase in the first $5 \mathrm{~h}$ and were subsequently reduced at $18 \mathrm{~h}$, followed by a stable level between 18 and $48 \mathrm{~h}$. Furthermore, AADC levels within $48 \mathrm{~h}$ were elevated compared with the control group, while COMT levels showed a significant reduction between 1 and $48 \mathrm{~h}$ (Fig. 3B).

Correlation between DA and 5-HT levels in the plasma, hippocampus, cortex and striatum following the co-administration. In order to investigate the association between the neurotransmitters following the co-administration of $\beta$-asarone and L-dopa, the correlations between DA and 5-HT in the cortex, striatum, hippocampus and plasma were analyzed using 
Table I. Correlation between DA and 5-HT in rat plasma and brain tissues within $48 \mathrm{~h}$.

r-value

\begin{tabular}{llccc}
\cline { 2 - 5 } Group & Striatum & Cortex & Hippocampus & Plasma \\
\hline $1 \mathrm{~h}$ & 0.289 & 0.871 & 0.501 & 0.214 \\
$5 \mathrm{~h}$ & $0.961^{\mathrm{a}}$ & 0.178 & 0.831 & 0.182 \\
$18 \mathrm{~h}$ & 0.296 & 0.700 & $0.976^{\mathrm{a}}$ & $0.989^{\mathrm{a}}$ \\
$48 \mathrm{~h}$ & 0.579 & 0.692 & $0.965^{\mathrm{a}}$ & 0.944
\end{tabular}

Pearson correlation analysis demonstrated that there were significant positive correlations between DA and 5-HT in the plasma, striatum, hippocampus and cortex. Data represent the mean \pm standard deviation of 8 rats. ${ }^{a} \mathrm{P}<0.05$, vs. control group. DA, dopamine; 5-HT, serotonin.

Pearson correlation analysis (Table I). The correlation between DA and 5-HT was positive and statistically significant in the striatum at $5 \mathrm{~h}(\mathrm{r}=0.961, \mathrm{P}<0.05)$. In addition, the correlations between DA and 5-HT in the hippocampus at 18 and $48 \mathrm{~h}$ were 0.976 and 0.965 , respectively $(\mathrm{P}<0.05)$. Furthermore, the correlation between DA and 5-HT in the plasma at $18 \mathrm{~h}$ was $0.989(\mathrm{P}<0.05)$. The results indicated that there were significant positive correlations between DA and 5-HT in the striatum at $5 \mathrm{~h}$, in the hippocampus at 18 and $48 \mathrm{~h}$ and in the plasma at $18 \mathrm{~h}$.

\section{Discussion}

$\beta$-asarone is a major component of Acorus tatarinowii Schott, and has a significant pharmacological effect in attenuating neuronal apoptosis, thus protecting against neurotoxicity (16). Furthermore, L-dopa remains the most effective medicine for the treatment of PD (7). To date, researchers have combined L-dopa with other medicines, such as carbidopa, for the treatment of PD, in order to mitigate the side-effects associated with L-dopa $(17,18)$; however, such combinations have not resolved the adverse reactions that result from chronic use of L-dopa. Notably, we demonstrated in a previous study that $\beta$-asarone and L-dopa co-administration is able to significantly increase the striatal dopamine (DA) levels in healthy rats (11). However, the dynamic changes in the levels of DA, L-dopa, DOPAC, HVA and 5-HT in the plasma and brain of healthy rats within $48 \mathrm{~h}$ of the co-administration treatment remain unknown. Therefore, a quantitative HPLC method was employed to analyze the levels of these five neurotransmitters in the striatum, hippocampus, cortex and plasma of rats at 1 , 5,18 and $48 \mathrm{~h}$ following treatment. In addition, we determined the dynamic change in a number of enzymes associated with these neurotransmitters in the plasma and striatum within $48 \mathrm{~h}$.

In the present study, it was observed that the striatal levels of L-dopa, DA, DOPAC and HVA increased after the co-administration and peaked at $1 \mathrm{~h}$, followed by reduction or return to the normal levels between 5 and 48 h. Notably, DA metabolism occurs primarily in the striatum (19). The present results suggested that striatal DA levels were increased compared with those in the hippocampus, cortex and plasma within $48 \mathrm{~h}$, indicating that the co-administration may aid in maintaining DA levels in the striatum.

Compared with the control group, the DA/L-dopa ratio in the striatum exhibited a rapid increase and peaked within the initial $5 \mathrm{~h}$ after treatment, followed by a sharp decline at $18 \mathrm{~h}$, and subsequent increase. In addition, the DA/L-dopa ratio in the striatum was higher compared with that in the hippocampus and cortex between 1 and $48 \mathrm{~h}$. These results indicated that the transformation of L-dopa to DA occurred primarily in the striatum after co-administration. In addition, the (DOPAC + HVA)/DA ratios in the striatum, hippocampus and cortex exhibited a significant reduction compared with the control group. These results indicated that the co-administration of $\beta$-asarone and L-dopa may be able to reduce the metabolism of DA.

In order to identify the mechanism through which DA levels are elevated predominantly in the striatum, the functions of TH, AADC, COMT and MAO-B were investigated. $\mathrm{TH}$ is the primary regulator and rate-limiting enzyme of L-dopa synthesis (3). The results of the present study showed that striatal $\mathrm{TH}$ levels increased and peaked at $1 \mathrm{~h}$, and subsequently returned to the normal levels at $5 \mathrm{~h}$. This may explain why L-dopa level at in the striatum were higher at $1 \mathrm{~h}$ compared with at 5-48 h. In addition, L-dopa is known to be converted into DA by AADC (4). The current results indicated that striatal AADC levels in the treatment groups increased within $48 \mathrm{~h}$ compared with the control group, which was consistent with the observation that striatal DA levels within $48 \mathrm{~h}$ were elevated compared with levels in the hippocampus and cortex. Furthermore, DA may be degraded into DOPAC by MAO-B and into HVA by COMT $(5,6)$. In the present study, striatal levels of MAO-B and COMT in the treatment groups were reduced compared with the control group, which may explain why the (DOPAC + HVA)/DA ratio in the striatum was reduced compared with the control group during $48 \mathrm{~h}$.

In the plasma, the levels of L-dopa, DA, DOPAC and HVA increased and peaked at $1 \mathrm{~h}$ after the co-administration, and then showed a sharp decrease between 5 and $48 \mathrm{~h}$. Based on the ratios of DA/L-dopa and (DOPAC + HVA)/DA, the turnover rate of L-dopa was found to peak at $5 \mathrm{~h}$ after co-administration, followed by a rapid decline between 5 and $48 \mathrm{~h}$. However, the turnover rate of DA exhibited a sharp decrease between 1 and $48 \mathrm{~h}$ compared with control group. In addition, TH levels increased and peaked at $1 \mathrm{~h}$ prior to returning to the normal levels. AADC levels peaked at $5 \mathrm{~h}$, and were higher compared with the control group. In addition, COMT levels exhibited a decreasing trend within $48 \mathrm{~h}$ compared with the control group. Thus, collectively the present results suggest that the co-administration may promote the generation of DA by enhancing the activity of AADC, in addition to reducing the metabolism of DA by inhibiting the activity of COMT.

In the present study, the 5-HT levels in the plasma, striatum, hippocampus and cortex of the rats exhibited no statistically significant differences when compared with the control group rats. However, the correlation between DA and 5-HT was positive and significant in the striatum, cortex, hippocampus and plasma at 5, 1, 18-48 and $18 \mathrm{~h}$, respectively. The results indicated that the statistically significant correlation identified between DA and 5-HT was associated with the brain and plasma areas in healthy rats following the co-administration. 
In conclusion, the present study reported the dynamic changes in the levels of L-dopa, DA, DOPAC, HVA and 5-HT in the striatum, cortex, hippocampus and plasma of rats within $48 \mathrm{~h}$ of the co-administration of $\beta$-asarone and L-dopa. Notably, the co-administration exerted the effect of maintaining a steady DA level in the striatum during the 48 -h period. Furthermore, the co-administration appeared to promote the generation of DA by enhancing the activity of AADC, while reducing the metabolism of DA by inhibiting the activity of COMT. Therefore, the co-administration of $\beta$-asarone and L-dopa may provide a beneficial intervention for the treatment of PD.

\section{Acknowledgements}

This study was supported by grants from the Guangdong Natural Science Foundation of China (No. S2012010010625) and the First Clinical Medical College of Guangzhou University of Chinese Medicine Excellence Doctoral Dissertation Cultivation Project (No. YB201403).

\section{References}

1. Nutt JG and Wooten GF: Clinical practice. Diagnosis and initial management of Parkinson's disease. N Engl J Med 353: 1021-1027, 2005.

2. Calabresi P, Giacomini P, Centonze D and Bernardi G: Levodopa-induced dyskinesia: A pathological form of striatal synaptic plasticity? Ann Neurol 47: S60-S68, 2000.

3. Baier CJ,Pallarés ME, AdroverE,Katunar MR,Raisman-Vozari R and Antonelli MC: Intrastriatal 6-OHDA lesion differentially affects dopaminergic neurons in the ventral tegmental area of prenatally stressed rats. Neurotox Res 26: 274-284, 2014.

4. Ren J, Zhang Y, Jin H, Yu J, Zhou Y, Wu F and Zhang W: Novel inhibitors of human DOPA decarboxylase extracted from Euonymus glabra Roxb. ACS Chem Biol 9: 897-903, 2014.

5. Bolea I, Colivicchi MA, Ballini C, Marco-Contelles J, Tipton KF Unzeta $\mathrm{M}$ and Della Corte L: Neuroprotective effects of the MAO-B inhibitor, PF9601N, in an in vivo model of excitotoxicity. CNS Neurosci Ther 20: 641-650, 2014.

6. Onzawa Y, Kimura Y, Uzuhashi K, Shirasuna M, Hirosawa T, Taogoshi $\mathrm{T}$ and Kihira K: Effects of 3-O-methyldopa, L-3,4-dihydroxyphenylalanine metabolite, on locomotor activity and dopamine turnover in rats. Biol Pharm Bull 35: 1244-1248, 2012
7. Noack C, Schroeder C, Heusser K and Lipp A: Cardiovascular effects of levodopa in Parkinson's disease. Parkinsonism Relat Disord 20: 815-818, 2014.

8. Hong JY, Oh JS, Lee I, Sunwoo MK, Ham JH, Lee JE, Sohn YH, Kim JS and Lee PH: Presynaptic dopamine depletion predicts levodopa-induced dyskinesia in de novo Parkinson disease. Neurology 82: 1597-1604, 2014.

9. Alachkar A, Brotchie JM and Jones OT: Locomotor response to L-DOPA in reserpine-treated rats following central inhibition of aromatic L-amino acid decarboxylase: Further evidence for non-dopaminergic actions of L-DOPA and its metabolites. Neurosci Res 68: 44-50, 2010.

10. Wu HB and Fang YQ: Pharmacokinetics of beta-asarone in rats Yao Xue Xue Bao 39: 836-838, 2004 (In Chinese).

11. Huang L, Deng M, Zhang S, Fang Y and Li L: Coadministration of $\beta$-asarone and levodopa increases dopamine in rat brain by accelerating transformation of levodopa: A different mechanism from Madopar. Clin Exp Pharmacol Physiol 41: 685-690. 2014

12. Veloso A, Fernández R, Astigarraga E, Barreda-Gómez G, Manuel I, Giralt MT, Ferrer I, Ochoa B, Rodríguez-Puertas R and Fernández JA: Distribution of lipids in human brain. Anal Bioanal Chem 401: 89-101, 2011.

13. Liu L and Fang YQ: Analysis of the distribution of $\beta$-asarone in rat hippocampus, brainstem, cortex and cerebellum with GC-MS. J Med Plants Res 9: 1728-1734, 2011.

14. Fang YQ, Shi C, Liu L and Fang RM: Analysis of transformation and excretion of $\beta$-asarone in rabbits with GC-MS. Eur J Drug Metab Pharmacokinet 37: 187-190, 2012.

15. Zhang S, Gui XH, Xue ZF, Huang LP, Fang RM, Ke XH, Li L and Fang YQ: Dynamic of neurochemical alterations in striatum, hippocampus and cortex after the 6-OHDA mesostriatal lesion. Int J Dev Neurosci 36: 32-37. 2014;

16. Wei G, Chen YB, Chen DF, Lai XP, Liu DH, Deng RD, Zhou JH, Zhang SX, Li YW, Lii H, et al: $\beta$-Asarone inhibits neuronal apoptosis via the $\mathrm{CaMKII/CREB/Bcl-2} \mathrm{signaling} \mathrm{pathway} \mathrm{in}$ an in vitro model and A $\beta$ PP/PS1 mice. J Alzheimers Dis 33: 863-880, 2013.

17. Takáts A, Nagy H, Radics P, Tóth A and Tamás G: Treatment possibilities in advanced Parkinson's disease. Ideggyogy Sz 66: 365-371, 2013 (In Hungarian).

18. Lazzara CA, Riley RR, Rane A, Andersen JK and Kim YH: The combination of lithium and I-Dopa/Carbidopa reduces MPTPinduced abnormal involuntary movements (AIMs) via calpain-1 inhibition in a mouse model: Relevance for Parkinson's disease therapy. Brain Res: June 26, 2015 (epub ahead of print).

19. Wu T, Liu J, Zhang H, Hallett M, Zheng Z and Chan P: Attention to automatic movements in Parkinson's disease: Modified automatic mode in the striatum. Cereb Cortex: June 12, 2014 (epub ahead of print). 